\title{
LETTERS
}

\section{The future of electronic medical records in Canada}

What can the wealthiest person in the world teach Canada about the design of electronic medical record (EMR) systems?

A lot.

Around 2002, when Amazon was still more or less an online bookstore squeezed by shrinking margins, Jeff Bezos issued a single mandate via email (www.businessinsider.com/steve-yegge -google-platform-rant-2011-10) that set Amazon on the path to becoming the most dominant online retailing platform in the world. The keyword here is "platform": Amazon doesn't sell everything itself, but it provides the interface between buyers and sellers. In just one email, Jeff Bezos changed the culture of the company from one focused on products to one focused on services. In doing so, he also made the company vastly more efficient, leveraged the ingenuity of third-party developers and turned cost items into revenue streams.

How did he do it? And what can we learn from this case to improve the efficiency of our health care system?

Essentially, the genius of the mandate was to make all the teams of programmers working for Amazon communicate via the same interface, and to make the internal services that the teams provide to each other accessible to third-party programmers outside the company at the flip of a switch. In this way, Bezos drastically reduced the amount of redundant work the independent teams had to do. Instead of independently building a particular software tool, engineers could just look for it in Amazon's directory of services and rent it out - chances are someone has done it already. The fundamental resources that Amazon programmers needed - such as computing power, online storage, database management, data analytics - were now services that could be offered to the outside world for profit. In 2017, this collection of online services, known as Amazon Web Services (AWS), generated revenues of \$US17.4 billion, some $10 \%$ of Amazon's total. AWS remains the dominant player in cloud computing, taking more market share than Microsoft, Google and IBM combined. ${ }^{1}$ Before the famous Bezos mandate, none of this would have been possible: the massive amounts of computational resources that Amazon consumed would have constituted purely an expense on the balance sheet.

There are obvious parallels to be drawn between Amazon of the early 2000s and Canada's health care system. Instead of teams of programmers, we have teams of physicians. Instead of a standardized communication interface, we have a balkanized system of EMRs, none of which communicate with each other. Instead of asking a colleague to fax over a patient's test results, it's often easier just to repeat the investigation. In an era of nonstop hand-wringing about ballooning health care costs, this strikes me as irresponsible at best.

The Ontario EHealth debacle ${ }^{2}$ is a prime example of how a focus on the perfect product (in this context, the perfect EMR) is doomed to fail. There is simply no way to predict and satisfy the needs of all stakeholders: our needs are too disparate. The path to success lies in establishing a set of standards to which all EMRs must adhere, and opening the market to the ingenuity of third- party developers to cater to the specific needs and whims of each customer, all while maintaining interoperability.

In 1983, Pierre Trudeau introduced the Canada Health Act, which enshrined portability as a central tenet of our delivery of health care. While the letter of the law focuses on financial portability, the current barriers to effective communication between providers in different provinces impede delivery of care to the extent that the idea of portability is infringed upon in spirit.

We need a mandate, from the federal government, that forces the provinces and the EMR providers to adhere to a common standard, not a single electronic health record as suggested by Dr. Nav Persaud in his editorial. ${ }^{3}$ This would go a long way toward decreasing the costs of repeated tests, reducing errors in communication between practitioners, and serving Canadians in the way the Canada Health Act intended.

\section{Eric J. Zhao BSc MASc}

Medical student, University of British Columbia, Vancouver, BC

- Cite as: CMAJ 2019 May 13;191:E542. doi: $10.1503 / \mathrm{cmaj} .71858$

Competing interests: None declared.

\section{References}

1. Rama G. Report: AWS market share Is triple Azure's. AWS Insider 2017 Aug. 1. Available: https:// awsinsider.net/articles/2017/08/01/aws-marketshare-3x-azure.aspx (accessed 2018 Mar. 30).

2. EHealth scandal a $\$ 1 B$ waste: auditor. $C B C$ News 2009 Oct. 7. Available: www.cbc.ca/news/ canada/toronto/ehealth-scandal-a-1b-wasteauditor-1.808640 (accessed 2018 Mar. 30).

3. Persaud N. A national electronic health record for primary care. CMAJ 2019;191:E28-9. 